\title{
EXISTENCE OF PERIODIC SOLUTIONS FOR NONLINEAR EVOLUTION EQUATIONS IN HILBERT SPACES
}

\author{
NORIMICHI HIRANO
}

(Communicated by Barbara Lee Keyfitz)

\begin{abstract}
In this paper, we consider the existence and multiplicity of periodic solutions of the problem $u^{\prime}+A u \ni g(t, u)$ where $A$ is a subdifferential of a convex function defined in a Hilbert space $H$ and $g: R \times H \rightarrow H$ is a Carathéodory function periodic with respect to the first variable.
\end{abstract}

\section{INTRODUCTION}

Let $(H,\|\cdot\|)$ be a Hilbert space with an inner product $\langle\cdot, \cdot\rangle$ and $A \subset$ $H \times H$ be an m-accretive operator given by the form $A=\partial \varphi$, where $\partial \varphi$ is the subdifferential of a lower semicontinuous proper convex function $\varphi: D(\varphi) \subset$ $H \rightarrow R$, with domain $D(\varphi)$ dense in $H$. In the present paper, we consider the existence of T-periodic solutions for nonlinear evolution equations of the form

$$
\frac{d u}{d t}+A u \ni g(t, u), \quad t \in R,
$$

where $g: R \times H \rightarrow H$ is a Carathéodory function.

We state our assumptions imposed on $A$ and $g$ :

$\left(H_{1}\right)$ For some $\lambda>0, J_{\lambda}=\left(I+\lambda^{-1} A\right)^{-1}$ is a compact mapping on $H$;

$\left(H_{2}\right) \quad g: R \times H \rightarrow H$ is a Carathéodory mapping (i.e., for each $v \in H$ the mapping $t \rightarrow g(t, v)$ is measurable, and for each $t \in R$ the mapping $v \rightarrow g(t, v)$ is continuous) and satisfies that for some $M_{1}, M_{2}>0$

$$
\|g(t, v)\| \leq M_{1}\|v\|+M_{2} \quad \text { for all } t \in R \text { and } v \in H .
$$

We now state our main result.

Theorem. Suppose that $\left(H_{1}\right)$ and $\left(H_{2}\right)$ hold. Assume further that $g$ is $T$ periodic with respect to the first variable and satisfies that there exist positive constants $a$ and $b$ such that

$$
\langle z-g(t, v), v\rangle \geq a\|v\|^{2}-b \quad \text { for all } v \in D(A) \text { and } z \in A v \text {. }
$$

Then the problem $(\mathrm{P})$ has at least one T-periodic mild solution.

Received by the editors November 26, 1991 and, in revised form, March 2, 1992 and April 28, 1992.

1991 Mathematics Subject Classification. Primary 34G20, 34C25; Secondary 35K55.

Key words and phrases. Accretive operator, periodic solution, nonlinear evolution equations.

(C) 1993 American Mathematical Society $0002-9939 / 93 \$ 1.00+\$ .25$ per page 
Existence of T-periodic solutions for $(\mathrm{P})$ has been investigated by many authors (cf. [5-7]) under the assumption that $A$ is m-accretive and $g$ is Lipschitz continuous with respect to the second variable. In the case that $g$ is merely continuous with respect to the second variable, the existence of periodic solutions of $(\mathbf{P})$ was studied in $[2,8,9]$. Becker [2] considered the case that $g$ is continuous and $A: D(A) \rightarrow H$ is a closed densely defined linear operator. Recently, Vrabie [9] extended Becker's result to fully nonlinear cases. In [9] it is assumed that $H$ is a real Banach space and $A: D(A) \rightarrow 2^{H}$ is an m-accretive operator such that $\overline{D(A)}$ is convex, $A$ generates a compact semigroup,

$\left(c_{1}\right)$ there exists $a>0$ such that $A-a I$ is m-accretive,

$\left(c_{2}\right) g$ is a T-periodic Carathéodory mapping and satisfies

$$
\lim _{r \rightarrow \infty}(1 / r) \sup \{\|g(t, v)\|: t \in R, \quad v \in \overline{D(A)},\|v\| \leq r\}=m<a .
$$

For additional references for periodic solutions of the problem $(\mathrm{P})$, the reader is referred to Vrabie [9]. In the introduction of [9], the reader can find detailed explanations of the difficulty caused by the lack of Lipschitz continuity for $g$ and of the results established so far. Our approach is quite different from that employed in [9]. Though our method requires that $A$ is a subdifferential of a functional, we do not need that $A-a I$ is m-accretive for some $a>0$ and then our results can be applied to a wide class of elliptic operators. The assumption $(*)$ is a unilateral condition and then we do not need the opposite side restriction. (See §3.) We assume that the reader is familiar with the theory of nonlinear evolution equations. (See Barbu [1] and Brezis [4] for the basic concepts and results of nonlinear evolution equations.)

\section{Proof of TheOREM}

We denote by $L^{2}(0, T ; H)$ the space of functions $v:[0, T] \rightarrow H$ such that $\int_{0}^{T}\|v\|^{2} d t<\infty$. The norm and the inner product of $L^{2}(0, T ; H)$ are denoted by $\|\cdot\|_{T}$ and $\langle\langle\cdot, \cdot\rangle\rangle$, respectively. We identify the functions in $L^{2}(0, T ; H)$ with T-periodic functions. For each positive integer $m$, we denote by $W^{m, 2}(0, T ; H)$ the space of functions $v:[0, T] \rightarrow H$ such that $v^{(i)} \in L^{2}(0, T ; H)$ for $0 \leq i \leq m$, where $v^{(i)}$ denotes the $i$ th derivative in the sense of distribution. For a reflexive Banach space $E$, a mapping $T: E \rightarrow E^{*}$ is said to be pseudomonotone if $u_{n} \rightarrow u$ weakly in $E$ and lim $\sup _{n \rightarrow \infty}\left\langle T u_{n}, u_{n}-u\right\rangle \leq 0$ imply that $T u_{n} \rightarrow T u$ weakly in $E^{*}$ and

$$
\langle T u, u-v\rangle \leq \liminf _{n \rightarrow \infty}\left\langle T u_{n}, u_{n}-v\right\rangle \quad \text { for all } v \in E .
$$

We put $A_{\lambda}=\lambda\left(I-J_{\lambda}\right)$ for $\lambda>0$. It is known that $J_{\lambda}$ is nonexpansive (i.e., $\left\|J_{\lambda} x-J_{\lambda} y\right\| \leq\|x-y\|$ for $\left.x, y \in H\right)$. It is also known that $A_{\lambda} x \in A J_{\lambda} x$ and $J_{\lambda} x=J_{\mu}\left(J_{\lambda} x+\mu^{-1} A_{\lambda} x\right)$ for $\lambda, \mu>0$ and $x \in H$ (cf. [4]). It follows from this equality that $\left(H_{1}\right)$ implies that $J_{\lambda}$ is compact for all $\lambda>0$. For each $n \geq 1$, we put $A_{n}=\partial \varphi_{n}$, where $\varphi_{n}$ is a functional defined by $\varphi_{n}(x)=$ $\inf _{u \in H}\left\{n\|x-u\|^{2} / 2+\varphi(u)\right\}$. Since $\varphi$ is a proper convex lower semicontinuous function, the induced functional defined by $\tilde{\varphi}(u)(t)=\varphi(u(t))$ for $u \in L^{2}(0, T ; H)$ is proper convex lower semicontinuous and the domain $D(\tilde{\varphi})$ is dense in $L^{2}(0, T ; H)$. 
Therefore, $\tilde{A}=\partial \tilde{\varphi}$ is maximal monotone in $L^{2}(0, T ; H)$. For simplicity, we write $A$ and $\varphi$ instead $\tilde{A}$ and $\tilde{\varphi}$, respectively. In the following, we assume that $\left(H_{1}\right)$ and $\left(H_{2}\right)$ hold.

To find a solution of the problem $(\mathrm{P})$, we consider the approximate equations

$$
\left\{\begin{array}{l}
-\frac{1}{n} \frac{d^{2} u}{d t^{2}}+\frac{d u}{d t}+A_{n} u=g\left(t, J_{n} u\right), \\
u(T+t)=u(t), \quad t \in R .
\end{array}\right.
$$

Definition 1. A strong solution of the problem $\left(\mathrm{P}_{n}\right)$ is a T-periodic function $u: R \rightarrow H$ whose restriction to $[0, T]$ belongs to $W^{2,2}(0, T ; H)$ and which satisfies $\left(\mathrm{P}_{n}\right)$ a.e. for $t \in R$.

Definition 2. A generalized solution of $\left(\mathbf{P}_{n}\right)$, or a solution of $\left(\widetilde{\mathbf{P}}_{n}\right)$, is a Tperiodic function $u: R \rightarrow H$ whose restriction to $[0, T]$ belongs to $W^{1,2}(0, T ; H)$ and satisfies

$\left(\widetilde{\mathbf{P}}_{n}\right) \quad\left\langle\left\langle\frac{1}{n} \frac{d u}{d t}, \frac{d v}{d t}\right\rangle\right\rangle+\left\langle\left\langle\frac{d u}{d t}+A_{n} u-g\left(t, J_{n} u\right), v\right\rangle\right\rangle=0$

for all $v \in W$ verifying $v(0)=v(T)$.

In the following, we denote by $W$ the space defined by $W=\left\{v ; v: R \rightarrow H, v(t+T)=v(t)\right.$ for $t \in R$, and $\left.\left.v\right|_{[0, T]} \in W^{2,2}(0, T ; H)\right\}$ endowed with the norm $\|\cdot\|_{1, T}$ of $W^{1,2}(0, T ; H)$. That is,

$$
\|v\|_{1, T}^{2}=\left\|v_{t}\right\|_{T}^{2}+\|v\|_{T}^{2} \text { for } v \in W .
$$

Here we define an operator $T_{n}: W \rightarrow W^{*}$ by

$$
\begin{aligned}
\left\langle\left\langle T_{n} u, v\right\rangle\right\rangle= & \left\langle\left\langle\frac{1}{n} \frac{d u}{d t}, \frac{d v}{d t}\right\rangle\right\rangle \\
& +\left\langle\left\langle\frac{d u}{d t}+A_{n} u-g\left(t, J_{n} u\right), v\right\rangle\right\rangle \text { for } u, v \in W .
\end{aligned}
$$

Then it is obvious that if $u \in W$ satisfies $T_{n} u=0$, then $u$ is a solution of the problem $\left(\widetilde{\mathbf{P}}_{n}\right)$. It also follows from the definitions above that each solution $u \in W$ of $\left(\widetilde{\mathrm{P}}_{n}\right)$ is a solution of $\left(\mathrm{P}_{n}\right)$ if $\left.u\right|_{[0, T]} \in W^{2,2}(0, T ; H)$.

Remark 1 . The idea of considering approximating equations $\left(\mathbf{P}_{n}\right)$ is suggested by the observation that for each $n \geq 1$ the critical points of the functional $F_{n}$ defined by

$$
F_{n}(u)=\int_{0}^{T}\left(e^{-n t}\left(\frac{1}{2 n}\left\|u_{t}\right\|^{2}+\varphi(u)\right)\right) d t-\int_{0}^{T} e^{-n t} \int_{\Omega} \int^{u(t, x)} g(\tau) d \tau d x d t
$$

are solutions of the problem $\left(\mathbf{P}_{n}\right)$ provided that each critical point is contained in $W^{2,2}(0, T ; H)$. The existence of the critical points of $F_{n}$ follows easily from the fact that $F_{n}(v) \rightarrow \infty$, as $\|v\|_{1, T} \rightarrow \infty$ under the assumption $(*)$.

Remark 2. We may assume without any loss of generality that $\varphi$ attains its minimum at 0 . In fact, if $\varphi$ attains its minimum at $u_{0} \neq 0$, we put $\widetilde{A}(v)=$ $A\left(v+u_{0}\right)$ and $\tilde{g}(v)=g\left(v+u_{0}\right)$. Then if $\tilde{u}$ is a solution of the problem (P) with $A$ and $g$ replaced by $\tilde{A}$ and $\tilde{g}$, we can see that $u=\tilde{u}+u_{0}$ is a solution of (P). In the following, we assume that 0 is the minimal point of $\varphi$. Then, since $J_{n}$ is nonexpansive and $J_{n} 0=0$ for $n \geq 1$, we have that $\left\|J_{n} v\right\| \leq\|v\|$ and $\left\|A_{n} v\right\| \leq 2 n\|v\|$ for $n \geq 1$ and $v \in H$. 
Lemma 1. For each $n \geq 1, T_{n}: W \rightarrow W^{*}$ is a pseudomonotone operator. Proof. Fix $n \geq 1$. Let $\left\{u_{i}\right\} \subset W$ be a sequence such that $u_{i} \rightarrow u \in W$ weakly in $W$ and

$$
\limsup _{i \rightarrow \infty}\left\langle\left\langle T_{n} u_{i}, u_{i}-u\right\rangle\right\rangle \leq 0 .
$$

Since $\left\{\left\|u_{i}\right\|_{1, T}\right\}$ is bounded we may assume that $u_{i} \rightarrow u \in L^{2}(0, T ; H)$ weakly in $L^{2}(0, T ; H)$ and $A_{n} u_{i} \rightarrow z$ weakly in $L^{2}(0, T ; H)$. It also follows that

$$
\sup \left\{\left\|u_{i}(t)\right\|: i \geq 1,0 \leq t \leq T\right\}<\infty .
$$

Since $J_{n}$ is compact, we have that $\left\{J_{n}\left(u_{i}(t)\right): i \geq 1,0 \leq t \leq T\right\}$ is relatively compact in $H$. Since $J_{n}$ is nonexpansive, it follows from the boundedness of $\left\{\left\|u_{i}\right\|_{1, T}\right\}$ that $\left\{\left(J_{n}\left(u_{i}\right)\right)_{t}: t \geq 1\right\}$ is bounded in $L^{2}(0, T ; H)$. Thus we obtain that $\left\{J_{n}\left(u_{i}\right)\right\}$ is relatively compact in $L^{2}(0, T ; H)$. Then we may assume by extracting subsequences that $J_{n}\left(u_{i}\right)$ converges to $v \in L^{2}(0, T ; H)$ strongly in $L^{2}(0, T ; H)$. Since $u_{i} \rightarrow u$ weakly in $L^{2}(0, T ; H)$, we have that $v=J_{n} u$ (cf. [4, Proposition 2.5]). Then it follows that $g\left(J_{n}\left(u_{i}\right)\right) \rightarrow g\left(J_{n}(u)\right)$ strongly in $L^{2}(0, T ; H)$. Therefore, we have

$$
\limsup _{i \rightarrow \infty} \int_{0}^{T}\left(\frac{1}{n}\left\langle u_{i t}, u_{i t}-u_{t}\right\rangle-\left\langle u_{i t}, u\right\rangle+\left\langle A_{n} u_{i}, u_{i}-u\right\rangle\right) d t \leq 0 .
$$

Noting that

$$
\left\|u_{t}\right\|_{T} \leq \liminf _{i \rightarrow \infty}\left\|u_{i t}\right\|_{T}
$$

and

$$
\lim _{n \rightarrow \infty}\left\langle\left\langle u_{i t}, u\right\rangle\right\rangle=\lim _{n \rightarrow \infty}\left\langle\left\langle u_{i}, u_{t}\right\rangle\right\rangle=0,
$$

we find that $\lim \sup _{i \rightarrow \infty}\left\langle\left\langle A_{n} u_{i}, u_{i}-u\right\rangle\right\rangle \leq 0$. Then, since $A_{n}$ is maximal monotone, we have from [1, Chapter II, Lemma 1.3] that $\lim _{i \rightarrow \infty}\left\langle\left\langle A_{n} u_{i}, u_{i}\right\rangle\right\rangle=$ $\left\langle\left\langle A_{n} u, u\right\rangle\right\rangle$ and $A_{n} u_{i}$ converges weakly to $A_{n} u$. Then we obtain that $T_{n} u_{i}$ converges weakly to $T_{n} u$ and then $\left\langle\left\langle T_{n} u, u-v\right\rangle\right\rangle \leq \liminf _{i \rightarrow \infty}\left\langle\left\langle T_{n} u_{i}, u_{i}-v\right\rangle\right\rangle$ for all $v \in W$. This completes the proof.

Lemma 2. There exists $n_{0} \geq 1$ such that for each $n \geq n_{0}$ the mapping $T_{n}$ satisfies

$$
\lim _{\|v\|_{1}, T^{\rightarrow}}\left\langle\left\langle T_{n} v, v\right\rangle\right\rangle=\infty
$$

Proof. From the definition of $A_{n}$, we have that

$$
\left\langle A_{n} v, v\right\rangle=n\left\|v-J_{n} v\right\|^{2}+\left\langle A_{n} v, J_{n} v\right\rangle \text { for } v \in H .
$$

Then we have by $(*)$ that for each $n \geq 1$ and $v \in H$

$$
\begin{aligned}
\left\langle A_{n} v-\right. & \left.g\left(t, J_{n} v\right), v\right\rangle \\
\geq & \left\langle A_{n} v-g\left(t, J_{n} v\right), J_{n} v\right\rangle+n\left\|v-J_{n} v\right\|^{2} \\
& -\left(M_{1}\left\|J_{n} v\right\|+M_{2}\right)\left\|v-J_{n} v\right\| \\
\geq & a\left\|J_{n} v\right\|^{2}+n\left\|v-J_{n} v\right\|^{2}-\left(M_{1}\left\|J_{n} v\right\|+M_{2}\right)\left\|v-J_{n} v\right\|-b .
\end{aligned}
$$

Let $m \geq 1$ satisfy

$$
c=a\left(\frac{m-1}{m}\right)^{2}-\frac{M_{1}}{m}>0 .
$$


If $v \in H$ satisfies $\left\|v-J_{n} v\right\| \leq(1 / m)\|v\|$, then, recalling that $\left\|J_{n} v\right\| \leq\|v\|$, we have by (2.4) and (2.5) that

$$
\begin{aligned}
\left\langle A_{n} v-g\left(t, J_{n} v\right), v\right\rangle & \geq a\left(\frac{m-1}{m}\right)^{2}\|v\|^{2}-\frac{M_{1}}{m}\|v\|^{2}-\frac{M_{2}}{m}\|v\|-b \\
& =c\|v\|^{2}-\frac{M-2}{m}\|v\|-b .
\end{aligned}
$$

On the other hand, for $v \in H$ satisfying $\left\|v-J_{n} v\right\|>(1 / m)\|v\|$, we find by (2.4) that

$$
\left\langle A_{n} v-g\left(t, J_{n} v\right), v\right\rangle \geq\left(\frac{n}{m^{2}}-2 M_{1}\right)\|v\|^{2}-2 M_{2}\|v\|-b
$$

Then combining (2.6) and (2.7), we find that there exist $n_{0} \geq 1, C \geq 0$, and $\rho>0$ such that for each $n \geq n_{0}$

$$
\left\langle A_{n} v-g\left(t, J_{n} v\right), v\right\rangle \geq \rho\|v\|^{2}-C \text { for all } t \in R \text { and } v \in H .
$$

Now let $n \geq n_{0}$. Then for each $v \in W$ we have by (2.8) and the definition of $T_{n}$ that

$$
\left\langle\left\langle T_{n} v, v\right\rangle\right\rangle \geq \int_{0}^{T}\left(\frac{1}{n}\left\|v_{t}\right\|^{2}+\rho\|v\|^{2}-C\right) d t .
$$

This implies that $\lim _{\|v\|_{1, T} \rightarrow \infty}\left\langle\left\langle T_{n} v, v\right\rangle\right\rangle=\infty$.

Lemma 3. For each $n \geq n_{0}$, there exists a strong solution $u_{n} \in W^{2,2}(0, T ; H)$ of the problem $\left(\mathbf{P}_{n}\right)$ satisfying

$$
\left\|\frac{1}{n} u_{n t t}\right\|_{T}^{2} \leq 4\left\|u_{n t}\right\|_{T}^{2}+2 M_{1}^{2}\left\|u_{n}\right\|_{T}^{2}+2 T M_{2}^{2} .
$$

Proof. Let $n \geq n_{0}$. Then we have by Lemma 2 that $T_{n}$ is coercive in $W$. Then, since $T_{n}$ is pseudomonotone, we have that there exists $u_{n} \in W$ such that $T_{n} u_{n}=0$ (cf. [3]). That is, $u_{n}$ is a solution of $\left(\widetilde{\mathbf{P}}_{n}\right)$. We show that $u_{n} \in W^{2,2}(0, T ; H)$. Let $\left\{u_{n}^{i}\right\} \subset W^{2,2}(0, T ; H) \cap W$ be an approximating sequence such that $u_{n}^{i}$ converges to $u_{n}$ strongly in $W^{1,2}(0, T ; H)$. Then from the definition of $T_{n}$ we have that for each $v \in W$

$$
\left\langle\left\langle-\frac{1}{n} u_{n t t}^{i}+u_{n t}^{i}+A_{n} u_{n}^{i}-g\left(t, J_{n} u_{n}^{i}\right), v\right\rangle\right\rangle \rightarrow 0 \quad \text { as } i \rightarrow \infty .
$$

This implies that $(1 / n) u_{n t t}^{i}$ converges to $u_{n t}+A_{n} u_{n}-g\left(t, J_{n} u_{n}\right)$ weakly in $W^{*}$. Since $u_{n t}+A_{n} u_{n}-g\left(t, J_{n} u_{n}\right) \in L^{2}(0, T ; H)$, we obtain that $u_{n t t} \in$ $L^{2}(0, T ; H)$. That is, $u_{n} \in W^{2,2}(0, T ; H)$ and

$$
-\frac{1}{n} u_{n t t}+u_{n t}+A_{n} u_{n}-g\left(t, J_{n} u_{n}\right)=0 .
$$

We multiply $(2.10)$ by $u_{n t t}$ and integrate over $[0, T]$. Then, noting that $A_{n}$ is Lipschitz continuous with Lipschitz constant $2 n$, we find

$$
\begin{aligned}
\int_{0}^{T} \frac{1}{n}\left\|u_{n t t}\right\|^{2} d t & \leq \int_{0}^{T}\left(\left\|u_{n t}\right\|\left\|\left(A_{n} u_{n}\right)_{t}\right\|+\left\|u_{n t t}\right\|\left(M_{1}\left\|J_{n} u_{n}\right\|+M_{2}\right)\right) d t \\
& \leq \int_{0}^{T}\left(2 n\left\|u_{n t}\right\|^{2}+\left\|u_{n t t}\right\|\left(M_{1}\left\|u_{n}\right\|+M_{2}\right)\right) d t
\end{aligned}
$$


Then we find

$$
\begin{aligned}
\int_{0}^{T}\left\|\frac{1}{n} u_{n t t}\right\|^{2} d t & \leq \int_{0}^{T}\left(2\left\|u_{n t}\right\|^{2}+\left\|\frac{1}{n} u_{n t t}\right\|\left(M_{1}\left\|u_{n}\right\|+M_{2}\right)\right) d t \\
& \leq 2\left\|u_{n t}\right\|_{T}^{2}+\frac{1}{2}\left\|\frac{1}{n} u_{n t t}\right\|_{T}^{2}+M_{1}^{2}\left\|u_{n}\right\|_{T}^{2}+T M_{2}^{2} .
\end{aligned}
$$

Then we can see that (2.9) follows.

Proof of Theorem. By Lemma 3, we have that for each $n \geq n_{0}$ there exists a strong solution $u_{n}$ of the problem $\left(\mathrm{P}_{n}\right)$. That is, $u_{n}$ is T-periodic and satisfies

$$
-\frac{1}{n} \frac{d^{2} u_{n}}{d t^{2}}+\frac{d u_{n}}{d t}+A_{n} u_{n}=g\left(t, J_{n} u_{n}\right) \text { a.e. } t \in R \text {. }
$$

We multiply $(2.11)$ by $u_{n}$ and integrate over $[0, T]$. Then we have by $(2.8)$

$$
\int_{0}^{T}\left(\frac{1}{n}\left\|u_{n t}\right\|^{2}+\rho\left\|u_{n}\right\|^{2}\right) d t \leq T C .
$$

Then we have that $\left\{\left\|u_{n}\right\|_{T}\right\}$ is bounded and that $\left\{\left\|g\left(t, J_{n} u_{n}\right)\right\|_{T}\right\}$ is bounded. We next multiply $(2.11)$ by $u_{n t}$ and integrate over $[0, T]$. Then, noting that

$$
\int_{0}^{T}\left\langle A_{n} u_{n}, u_{n t}\right\rangle d t=\varphi_{n}\left(u_{n}(T)\right)-\varphi_{n}\left(u_{n}(0)\right)=0
$$

we find

$$
\left\|u_{n t}\right\|_{T}^{2} \leq\left\|u_{n t}\right\|_{T}\left\|g\left(t, J_{n} u_{n}\right)\right\|_{T} \leq\left\|u_{n t}\right\|_{T}\left(M_{1}\left\|u_{n}\right\|_{T}+M_{2}\right)
$$

This implies that $\left\{\left\|u_{n t}\right\|_{T}\right\}$ is bounded. It then follows that

$$
\sup _{n \geq n_{0}}\left\|\left(J_{n} u_{n}\right)_{t}\right\|_{T}<\infty
$$

We also have by $(2.14)$ that

$$
\sup \left\{\left\|J_{n} u_{n}(t)\right\|: n \geq n_{0}, \quad 0 \leq t \leq T\right\}<\infty .
$$

On the other hand, recalling that (2.9) holds for all $n \geq n_{0}$, we have that $\left\{\left\|u_{n t t} / n\right\|\right\}$ is bounded. Then it follows from (2.11) that

$$
\sup _{n \geq n_{0}} \int_{0}^{T}\left\|A_{n} u_{n}\right\|^{2} d t<\infty .
$$

We now show that $\left\{J_{n} u_{n}\right\}$ is relatively compact in $L^{2}(0, T ; H)$. Let $\varepsilon>0$. Then, by (2.14) and (2.15), there exists an integer $m_{0}>0$ such that

$$
\left\|J_{n} u_{n}(t)-J_{n} u_{n}(s)\right\|^{2}<\varepsilon / 6 T \text { for all } n \geq n_{0} \text { and }|t-s|<2 T / m_{0} .
$$

On the other hand, we have that there exists $D>0$ such that $\inf \left\{\left\|A_{n} u_{n}(\tau)\right\|: t \leq \tau \leq t+T / m_{0}\right\}<D$ for all $n \geq n_{0}$ and $0 \leq t \leq T-T / m_{0}$.

We now choose $\left\{t_{m, n}: n_{0} \leq n, 1 \leq m \leq m_{0}\right\} \subset[0, T]$ such that

$$
T(m-1) / m_{0} \leq t_{m, n} \leq T m / m_{0} \text { and }\left\|A_{n} u_{n}\left(t_{m, n}\right)\right\| \leq D
$$

for $n \geq n_{0}$ and $1 \leq m \leq m_{0}$. Here we fix $n_{1} \geq 1$. Then, since

$$
J_{n}\left(u_{n}\left(t_{m, n}\right)\right)=J_{n_{1}}\left(J_{n} u_{n}\left(t_{m, n}\right)+A_{n} u_{n}\left(t_{m, n}\right) / n_{1}\right) \text { for } n \geq 1,
$$


we find by $\left(H_{1}\right)$ that $\left\{J_{n}\left(u_{n}\left(t_{m, n}\right)\right)\right\}$ is relatively compact. Then we may assume by extracting subsequences that $\left\{J_{n}\left(u_{n}\left(t_{m, n}\right)\right): n \geq 1\right\}$ is a convergent sequence for all $1 \leq m \leq m_{0}$. Then we have from (2.17) and the observation above that $\left\|J_{n}\left(u_{n}\right)-J_{n^{\prime}}\left(u_{n^{\prime}}\right)\right\|_{T}^{2}<\varepsilon$ for $n, n^{\prime}$ sufficiently large. Since $\varepsilon>0$ is arbitrary, we have that $\left\{J_{n}\left(u_{n}\right)\right\}$ is relatively compact in $L^{2}(0, T ; H)$. and

Therefore, we may assume that there exists a T-periodic function $u \in W$

$$
J_{n} u_{n} \rightarrow u \text { strongly in } L^{2}(0, T ; H) .
$$

Also we may assume that $A_{n} u_{n} \rightarrow z \in L^{2}(0, T ; H)$ weakly in $L^{2}(0, T ; H)$ by the boundedness of $\left\{\left\|A_{n} u_{n}\right\|_{T}\right\}$. On the other hand, we have

$$
\int_{0}^{T}\left\|u_{n}-J_{n} u_{n}\right\|^{2} d t=\frac{1}{n^{2}} \int_{0}^{T}\left\|A_{n} u_{n}\right\|^{2} d t \rightarrow 0 \quad \text { as } n \rightarrow \infty .
$$

Then we find by (2.18) and (2.19) that

$$
\lim _{m, n \rightarrow \infty} \int_{0}^{T}\left\langle A_{n} u_{n}-A_{m} u_{m}, u_{n}-u_{m}\right\rangle d t=0 .
$$

Since $A$ is maximal monotone in $L^{2}(0, T ; H)$, we have by [1, Chapter II, Proposition 1.1] that

$$
A_{n} u_{n} \rightarrow z \in A u \quad \text { weakly in } L^{2}(0, T ; H) .
$$

We also have that $\frac{1}{n} u_{n t t} \rightarrow 0$ weakly in $W^{1,2}(0, T ; H)$, because $\frac{1}{n} \int_{0}^{T}\left|u_{n t}\right|^{2} d t$ $\rightarrow 0$ as $n \rightarrow \infty$. Therefore, by (2.18), (2.19), and (2.20), we have that $u$ is a T-periodic solution of the problem $(\mathrm{P})$.

\section{EXAMPLE}

Let $\Omega \subseteq R^{N}$ be a bounded domain with a smooth boundary $\partial \Omega$ and $\psi: R \rightarrow R$ be a convex function. We put

$$
A u=\sum_{i=1}^{n} D_{i} a_{i}(D u) \text { for } u \in D(A)
$$

where $a_{i}(p)=\partial \psi(p) / \partial p_{i}$ for $p \in R^{N}$ and $D(A)=\left\{u \in H_{0}^{1}(\Omega): A u \in L^{2}(\Omega)\right\}$. We assume that $a_{i}$ is continuously differentiable and satisfies

$$
\sum_{i=1}^{n} a_{i}(p) p_{i} \geq \omega|p|^{2}-C_{0} \text { and } \sum_{i=1}^{n}\left|a_{i}(p)\right| \leq C_{1}|p|+C_{2}
$$

for all $p \in R^{N}$, where $C_{0}, C_{1}$, and $C_{2}$ are positive constants. Then $A$ is a subdifferential of the functional $u \rightarrow \int_{\Omega} \psi(u) d x$ and satisfies the assumption $\left(H_{1}\right)$ (cf. Vrabie [10]) with $H=L^{2}(\omega)$. Let $g: R \times R \rightarrow R$ be a Carathéodory mapping satisfying $g(t, x) x<\lambda_{1}(\omega-\varepsilon)|x|^{2}$ for some $\varepsilon>0$, where $\lambda_{1}$ is the first eigenvalue of $-\Delta$ under the Dirichlet boundary condition. Then, since

$$
\langle A u-g(t, u), u\rangle \geq \omega\|\nabla u\|^{2}-C_{0}-\lambda_{1}(\omega-\varepsilon)\|u\|^{2} \geq \lambda_{1} \varepsilon\|u\|^{2}-C_{0}
$$

for $u \in H_{0}^{1}(\omega)$, the condition $(*)$ is satisfied. We lastly see that the condition $(*)$ is strictly weaker than $\left(c_{1}\right)$ and $\left(c_{2}\right)$. Let $\psi(\tau)=\tau^{2}$ for $\tau \in R$ and 
$g(t, x)=\lambda_{1}(\sin t-2) \tau^{3} /\left(1+\tau^{2}\right)$ for $(t, \tau) \in R \times R$. Let $A$ be the operator defined by $(3.1)$ (i.e., $A=-\Delta)$. Then $\left(H_{1}\right),\left(H_{2}\right)$, and $(*)$ are satisfied. The operator $A$ satisfies $\left(c_{1}\right)$ for $a<\lambda_{1}$. But one can see that condition $\left(c_{2}\right)$ is not satisfied. Let $\psi(\tau)=\tau^{4} /\left(1+\tau^{2}\right)$ for $\tau \in R$ and $g$ be as above. In this case, $\left(H_{1}\right),\left(H_{2}\right)$, and $(*)$ are satisfied, but $A$ does not satisfy $\left(\mathrm{c}_{1}\right)$ for any $a>0$.

\section{ACKNOWLEDGMENT}

I am grateful to the referee for a careful reading of the paper and giving valuable suggestions.

\section{REFERENCES}

1. V. Barbu, Nonlinear semigroups and differential equations in Banach spaces, Editura Academiei, 1976.

2. R. I. Becker, Periodic solutions of semilinear equations of evolutions of compact type, J. Math. Anal. Appl. 82 (1981), 33-48.

3. H. Brézis, Equations et inequations nonlineaires dans des espace vectoriels en dualite, Ann. Inst. Fourier (Grenoble) 18 (1968), 115-175.

4. __ Operateurs maximaux monotones, North-Holland, Amsterdam, 1973.

5. F. E. Browder, Existence of periodic solutions for nonlinear equations of evolution, Proc. Nat. Acad. Sci. U.S.A. 53 (1965), 1100-1103.

6. J. H. Deimling, Periodic solutions of differential equations in Banach spaces, Manuscripta Math. 24 (1978), 31-44.

7. N. Hirano, Existence of multiple periodic solutions for a semilinear evolution equations, Proc. Amer. Math. Soc. 106 (1989), 107-114.

8. J. Prüss, Periodic solutions of semilinear evolution equations, Nonlinear Anal. 3 (1979), 221-235.

9. I. Vrabie, Periodic solutions for nonlinear evolution equations in a Banach space, Proc. Amer. Math. Soc. 109 (1990), 653-661.

10. __ Nonlinear version of Pazy's local existence theorem, Israel J. Math. 32 (1979), 221235.

Department of Mathematics, Faculty of Engineering, Yokohama National UniverSITy, 156 TokiwadaI, Hodogayaku, Yokohama 240, Japan

E-mail address: hirano@math.sci.ynu.ac.jp 